\title{
ACOLHIMENTO COM AVALIAÇÃO \\ E CLASSIFICAÇÃO DE RISCO: \\ ESTRUTURA E PROCESSO DO ATENDIMENTO
}

\author{
USER EMBRACEMENT WITH RISK ASSESSMENT \\ AND CLASSIFICATION: THE STRUCTURE \\ AND PROCEDURE OF ATTENDANCE
}

\section{ACOGIMIENTO CON EVALUACIÓN Y CLASIFICACIÓN DE RIESGOS: ESTRUCTURA Y PROCESO DE SERVICIO}

\author{
Ana Paula Santos de Jesus ${ }^{1}$ \\ Guiomar Rocha Pimentel Pimenta Rodrigues ${ }^{2}$ \\ Larissa Oliveira de Jesus ${ }^{3}$ \\ Meiry Fernanda Pinto Okuno ${ }^{4}$ \\ Ruth Ester Assayag Batista ${ }^{5}$
}

Como citar este artigo: Jesus APS, Rodrigues GRPP, Jesus LO, Okuno MFP, Batista REA. Acolhimento com avaliação e classificação de risco: estrutura e processo do atendimento. Rev baiana enferm. 2019;33:e28195.

\begin{abstract}
Objetivo: avaliar a estrutura e o processo do acolhimento com classificação de risco em uma unidade de emergência de acordo com as recomendações do Ministério da Saúde. Método: estudo descritivo e exploratório, realizado em uma unidade de emergência da Bahia. As técnicas de investigação foram a observação sistemática e a entrevista com enfermeiros. Para a análise foram feitas comparações e confrontações das avaliações das dimensões qualitativas de estrutura e processo. Resultados: os recursos disponíveis para o funcionamento da classificação de risco, quanto à estrutura física, recursos materiais e ao fluxo de atendimento contemplavam parcialmente o que é preconizado. Existiam lacunas, como sinalização dos espaços, ausência de alguns materiais na sala da classificação de risco, o não cumprimento do tempo de espera e ausência formal da referência/contrarreferência. Conclusão: a estrutura e o processo do acolhimento com classificação de risco em uma unidade de emergência precisavam de adequação do espaço físico e do suprimento total de recursos para cumprir o que é proposto pelo Ministério da Saúde.
\end{abstract}

Descritores: Enfermagem. Acolhimento. Triagem. Serviços Médicos de Emergência.

Objective: assessing the structure and the process of user embracement with risk classification in an emergency unit according to the recommendations of the Ministry of Health. Method: descriptive and exploratory study, carried out in an emergency unit in Bahia. The investigation techniques used were the systematic observation and the interview with nurses. Comparisons and confrontations of the evaluations of the qualitative dimensions of structure and procedure were used for analysis. Results: the resources available for the functioning of risk classification, with regards to physical structure, material resources, and the flow of attendance partially include the elements prescribed.

\footnotetext{
Enfermeira. Mestre em Enfermagem. Professora da Universidade Federal do Recôncavo da Bahia. Santo Antônio de Jesus, Bahia, Brasil.

Enfermeira. Especialista em Urgência e Emergência. São Paulo, SP, Brasil. guiomar.pimenta@outlook.com

Enfermeira. Especialista em Saúde Coletiva. Cruz das Almas, Bahia, Brasil.

Enfermeira. Doutora em Enfermagem. Professora da Universidade Federal de São Paulo. São Paulo, SP, Brasil.

Enfermeira. Pós-doutora em Enfermagem. Professora da Universidade Federal de São Paulo. São Paulo, SP, Brasil.
} 
There are shortcomings, such as the signaling of spaces, the absence of some materials in the risk classification room, the non-compliance with waiting times and the formal absence of references/counter-references. Conclusion: the structure and the procedure of user embracement with risk classification in an emergency unit need adjustments in its space and supplies, to be in accordance with the requirements of the Ministry of Health.

Descriptors: Nursing. User Embracement. Triage. Emergency Medical Services.

Objetivo: evaluarestructura yproceso de atención clasificadapor riesgo en unidad de urgencia según recomendaciones del Ministerio de Salud brasileño. Método: estudio descriptivo y exploratorio, en unidad de urgencia en Bahia, Brasil, cuyas técnicas de investigación fueron observación sistemática y entrevista con enfermeros. Para análisis, se realizaron comparaciones y confrontaciones de las evaluaciones de dimensiones cualitativas de estructura y proceso. Resultados: los recursos disponibles para funcionamiento de la clasificación de riesgos, con respecto a la estructura física, los recursos materiales y flujo de asistencia contemplan parcialmente lo que se recomienda. Hay lagunas, como señalización espacial, ausencia de materiales en la sala de clasificación de riesgos, incumplimiento del tiempo de espera y ausencia formal de referencia/contrarreferencia. Conclusión: estructura y proceso de atención clasificada por riesgo en unidad de urgencia necesitan espacio físico adecuado y suministro total de recursos para cumplir con lo que propone el Ministerio de Salud brasileño.

Descriptores: Enfermería. Acogimiento. Tamización. Servicios Médicos de Urgencia.

\section{Introdução}

O sistema de classificação de risco, também denominado de Acolhimento com Avaliação e Classificação de Risco (AACR), tem sido implantado gradativamente nos serviços de emergências do Brasil ${ }^{(1)}$. Atualmente, esta metodologia está incorporada tanto em unidades hospitalares de emergências quanto nas Unidades de Pronto Atendimento $^{(2)}$, frente à demanda excessiva por atendimento. A classificação de risco (CR) surgiu da necessidade da adoção de um critério para organização da demanda, não mais pela ordem de chegada, mas pela gravidade da situação clínica $^{(3-4)}$.

No Brasil, a superlotação dos Serviços de Emergência (SE) advém de múltiplos fatores: alta incidência e prevalência de doenças tropicais, como as arboviroses, transição no perfil epidemiológico da população e morbimortalidades relacionadas às doenças do aparelho circulatório, insuficiente estruturação da rede assistencial e aumento do número de acidentes automobilísticos e da violência urbana. Esses fatores têm contribuído para o abarrotamento do serviço e esta situação afeta de forma direta a qualidade da assistência prestada ao paciente ${ }^{(3,5)}$. Além disso, observa-se que esses serviços têm se tornado uma alternativa para o atendimento das doenças de baixa gravidade ${ }^{(5)}$.
Considerando o cenário e a complexidade da organização da rede de serviços públicos, o Programa Nacional de Avaliação de Serviços de Saúde tem incentivado a cultura avaliativa em estabelecimentos de saúde do Sistema Único de Saúde (SUS). Este programa estabelece dimensões para avaliações, que incluem a estrutura, o processo de trabalho, o resultado, a produção do cuidado, o gerenciamento dos riscos e a satisfação dos usuários em relação ao atendimento recebido $^{(6)}$.

Por outro lado, o AACR configura-se uma das principais intervenções potencialmente decisivas na reorganização dos serviços de emergência $^{(3)}$. Os componentes da sua estrutura, a identificação dos recursos físicos e materiais e o processo de atendimento aos usuários são áreas prioritárias para a realização de uma avaliação. Sendo assim, o estudo justifica-se pela necessidade de avaliação da qualidade desse serviço e por entender-se que este é o primeiro estudo desenvolvido para avaliá-lo em relação à qualidade da classificação de risco, após sua implantação. Além disso, pela necessidade de identificação de lacunas assistenciais e estabelecimento de estratégias de melhorias que garantam mais eficiência e eficácia no atendimento à urgência e emergência, proporcionando maior segurança 
para usuários e profissionais envolvidos nessa $\operatorname{atividade}^{(7)}$

Diante desse contexto, levantou-se o seguinte questionamento: Como é a estrutura e o processo de atendimento do AACR implantado em uma unidade de emergência? Está em conformidade com o preconizado pelo Ministério da Saúde (MS)? Para tanto, o objetivo deste estudo foi avaliar a estrutura e o processo do acolhimento com classificação de risco em uma unidade de emergência de acordo com as recomendações do Ministério da Saúde.

\section{Método}

Estudo exploratório, descritivo, realizado em uma unidade de emergência de um hospital geral, público, de alta complexidade, de Santo Antônio de Jesus, Bahia, que atende à demanda espontânea e referenciada dos 32 municípios circunvizinhos que compõem o Recôncavo baiano. As principais especialidades médicas oferecidas são: clínica médica, clínica pediátrica, cirurgia geral, ortopedia e traumatologia.

A unidade de emergência teve uma média de atendimentos mensal de 7.518 pacientes, considerando os meses de dezembro de 2014 a junho de 2015, período da coleta de dados. O serviço utilizava um protocolo institucional para a realização da CR. O modelo adotado era baseado na Cartilha de Acolhimento com Classificação de Risco proposta pelo MS e no Protocolo Estadual de Classificação de Risco da Secretaria de Saúde do Estado da Bahia ${ }^{(8)}$.

A técnica de investigação utilizada foi a observação sistemática, realizada nos turnos matutino, vespertino e noturno, com a utilização de um roteiro de observação previamente elaborado pelas pesquisadoras e adaptado do estudo de Mendes $^{(9)}$, contendo os seguintes elementos: acesso à informação, conforto, dignidade e cortesia, privacidade no atendimento, acolhimento e classificação de risco, priorização na atenção e assistência ao usuário. Além disso, foi realizada entrevista com enfermeiros do SE para auxiliar o preenchimento de um formulário estruturado, fundamentado nas recomendações do
$\mathrm{MS}^{(3)}$, contendo itens relativos à estrutura física, recursos materiais, além de outros, tais como: recursos humanos disponíveis no serviço de emergência (número de enfermeiros e técnicos de enfermagem), horário de funcionamento, classificação dos atendimentos, número de enfermeiros que atuam na classificação de risco, protocolo utilizado para classificação, insumos utilizados, tempo de espera para atendimento médico e tempo para reavaliação do paciente pelo enfermeiro.

Para organização, processamento, análise e interpretação das informações, foi feita uma abordagem baseada no estudo de $\operatorname{Mendes}^{(9)}$, a saber: o primeiro momento foi de valorização dos dados primários obtidos em cada um dos instrumentos; num segundo momento, a contextualização, comparação e confrontações dos dados, tendo como eixos centrais de análise a avaliação das dimensões qualitativas definidas pela pesquisa - avaliação da estrutura (acesso, conforto, dignidade e cortesia e recursos materiais) e avaliação do processo (privacidade no atendimento, acolhimento e classificação de risco, priorização na atenção ao usuário e assistência ao usuário).

Esta pesquisa foi aprovada pelo Comitê de Ética em Pesquisa (CEP) da Universidade Federal do Recôncavo da Bahia, sob o Parecer $n^{\circ}$ 773.010/14, e atendeu ao preconizado pela Resolução no 466/12 do Conselho Nacional de Saúde, sobre pesquisa com seres humanos.

\section{Resultados}

$\mathrm{Na}$ unidade de emergência avaliada, a implantação do AACR ocorreu em 2009, concomitantemente à abertura do hospital. Para atender à demanda de pacientes que procuravam esse serviço, a UE contava com 22 enfermeiros e 45 técnicos de enfermagem. Dentre os enfermeiros, cinco trabalhavam exclusivamente na CR. Esta funcionava em uma sala destinada para tal fim, nos três turnos do dia, de forma ininterrupta, com média diária de atendimentos de 250 pacientes. A priorização do atendimento ao usuário dava-se mediante a utilização de um protocolo 
próprio, que estratificava o risco em quatro níveis de prioridade: vermelho (emergência), amarelo (urgente), verde (pouco urgente) e azul (não urgente).

Os achados referentes à avaliação da estrutura constaram de atributos relacionados ao acesso à informação, conforto, dignidade e cortesia, e recursos materiais (Quadro 1). Vale ressaltar que a avaliação da estrutura do AACR englobou o setor interno da emergência e a área da recepção do hospital estudado. Quanto à avaliação da estrutura da unidade de emergência, pode ser observado que os recursos disponíveis para o funcionamento do AACR, no que tange à estrutura física e aos recursos materiais, foram contemplados parcialmente, considerando o que é preconizado pelo MS. Constatou-se que existem lacunas, como sinalização inadequada dos espaços e ausência de alguns materiais na sala da classificação de risco.

Quadro 1 - Síntese da avaliação da estrutura do Acolhimento com Avaliação e Classificação de Risco e da unidade de emergência estudada

\begin{tabular}{|c|c|}
\hline Dimensão avaliada & Resultados \\
\hline Acesso à informação & $\begin{array}{l}\text { Existiam placas indicativas dos espaços, mas não havia sinalização por } \\
\text { cores. }\end{array}$ \\
\hline \multirow[t]{5}{*}{ Conforto } & $\begin{array}{l}\text { O número de cadeiras para acomodação dos usuários e acompanhantes } \\
\text { era inferior à demanda; o mobiliário encontrava-se em bom estado de } \\
\text { conservação, limpo; e as macas foram encontradas com colchonete, } \\
\text { forradas e limpas. }\end{array}$ \\
\hline & $\begin{array}{l}\text { A limpeza dos espaços era satisfatória, exceto os banheiros da recepção, } \\
\text { que foram encontrados sujos e sem material de higiene. }\end{array}$ \\
\hline & $\begin{array}{l}\text { Existiam circuitos de ventilação com janelas; somente a sala de } \\
\text { reanimação era refrigerada. }\end{array}$ \\
\hline & A iluminação era adequada e segura ao usuário e ao profissional. \\
\hline & $\begin{array}{l}\text { Havia muitos ruídos desagradáveis, internos e externos, sobretudo durante } \\
\text { o dia. }\end{array}$ \\
\hline \multirow[t]{3}{*}{ Dignidade e Cortesia } & $\begin{array}{l}\text { Existia uma caixa de sugestões, reclamações e/ou dúvidas na recepção à } \\
\text { disposição do usuário. }\end{array}$ \\
\hline & $\begin{array}{l}\text { Não havia acomodação para os acompanhantes, exceto na sala do Serviço } \\
\text { Social e na ala pediátrica. }\end{array}$ \\
\hline & A entrada de acompanhantes era restrita às crianças e/ou idosos. \\
\hline Recursos materiais & $\begin{array}{l}\text { Os materiais monitor multiparamétrico, aparelho de Eletrocardiograma, } \\
\text { tábua rígida, bolsa-máscara-válvula (ambú) e medicamentos de uso } \\
\text { emergencial não foram encontrados na sala de Acolhimento com } \\
\text { Avaliação e Classificação de Risco. }\end{array}$ \\
\hline
\end{tabular}

Fonte: Elaboração própria.

A avaliação do processo de atendimento do AACR envolveu itens relacionados à privacidade no atendimento, funcionamento do AACR, priorização e assistência ao usuário. (Quadro 2). Os recursos disponíveis para o funcionamento da CR ficaram evidentes nas observações realizadas sobre o processo organizacional do AACR e nas respostas dos enfermeiros. No que tange ao fluxo de atendimento, também contemplavam parcialmente o preconizado pelo Ministério da
Saúde. Foram identificadas lacunas, como o não cumprimento do tempo estimado de espera, ausência formal da referência e contrarreferência. A CR era realizada por apenas um enfermeiro. A maioria relatou sobrecarga de trabalho na CR. O trabalho na CR consistia em realização da anamnese, exame físico sucinto, verificação dos sinais vitais, para posterior estratificação do risco do usuário, conforme o quadro clínico apresentado, encaminhando-o para o atendimento 
adequado. Além disso, destacava-se uma demanda elevada de pacientes que procuravam o serviço, dentre os quais aqueles com quadros não urgentes (azul), que são encaminhados ou orientados a procurar outros serviços.

Quadro 2 - Síntese dos elementos da avaliação do processo de atendimento do Acolhimento com Avaliação e Classificação de Risco

\begin{tabular}{|c|c|c|}
\hline $\begin{array}{l}\text { Dimensão } \\
\text { avaliada }\end{array}$ & Local avaliado & Resultados \\
\hline $\begin{array}{l}\text { Privacidade no } \\
\text { atendimento }\end{array}$ & $\begin{array}{l}\text { Sala de Classificação } \\
\text { de Risco e Unidade de } \\
\text { Emergência }\end{array}$ & $\begin{array}{l}\text { Quando examinado, o usuário, na maioria das } \\
\text { vezes, não foi exposto a outras pessoas que não os } \\
\text { profissionais de saúde. }\end{array}$ \\
\hline \multirow{4}{*}{$\begin{array}{l}\text { Acolhimento } \\
\text { com Avaliação e } \\
\text { Classificação de } \\
\text { Risco }\end{array}$} & $\begin{array}{l}\text { Recepção, Classificação } \\
\text { de Risco e Unidade de } \\
\text { Emergência }\end{array}$ & $\begin{array}{l}\text { Nem sempre a equipe multiprofissional estava } \\
\text { disponível para garantir a integralidade da assistência } \\
\text { ao usuário na chegada e durante sua permanência. }\end{array}$ \\
\hline & Recepção & $\begin{array}{l}\text { Os profissionais, na maioria das vezes, foram solícitos } \\
\text { para esclarecer dúvidas dos usuários de forma } \\
\text { humanizada. } \\
\text { Somente em alguns momentos foram fornecidas as } \\
\text { informações necessárias ao usuário no momento da } \\
\text { sua chegada e durante sua espera pelo atendimento. }\end{array}$ \\
\hline & $\begin{array}{l}\text { Classificação de Risco e } \\
\text { Unidade de Emergência }\end{array}$ & $\begin{array}{l}\text { Havia definição na prioridade do atendimento na } \\
\text { Classificação de Risco. }\end{array}$ \\
\hline & $\begin{array}{l}\text { Recepção e Classificação } \\
\text { de Risco }\end{array}$ & $\begin{array}{l}\text { Quando classificados como "azul”, os usuários eram } \\
\text { encaminhados a outros serviços. } \\
\text { Na maioria das vezes, não era feito o encaminhamento } \\
\text { formal (ficha de referência / contrarreferência). }\end{array}$ \\
\hline \multirow[t]{2}{*}{$\begin{array}{l}\text { Priorização } \\
\text { na atenção ao } \\
\text { usuário }\end{array}$} & $\begin{array}{l}\text { Recepção, Classificação } \\
\text { de Risco e Unidade de } \\
\text { Emergência }\end{array}$ & $\begin{array}{l}\text { O tempo de espera do usuário era mensurado na } \\
\text { chegada (preenchimento da ficha) até ser avaliado } \\
\text { pelo enfermeiro na Classificação de Risco; e após a } \\
\text { Classificação de Risco até o momento de ser atendido } \\
\text { pelo médico. } \\
\text { Os usuários eram comunicados sobre o tempo que } \\
\text { deveriam aguardar o atendimento. } \\
\text { Na maioria das vezes, os usuários não eram } \\
\text { comunicados, caso o tempo de espera extrapolasse. } \\
\text { Na maioria das vezes, os casos mais graves eram } \\
\text { priorizados. } \\
\text { Não havia reavaliação do usuário, caso o tempo de } \\
\text { espera extrapolasse o estimado. Se houvesse alguma } \\
\text { intercorrência, sim. }\end{array}$ \\
\hline & $\begin{array}{l}\text { Classificação de Risco e } \\
\text { Unidade de Emergência }\end{array}$ & $\begin{array}{l}\text { Todos os usuários passavam pela Classificação de } \\
\text { Risco, exceto as emergências (vermelho). } \\
\text { Havia um enfermeiro atuando diariamente na } \\
\text { Classificação de Risco. } \\
\text { O enfermeiro conseguia atender à demanda, mas } \\
\text { queixava-se da sobrecarga. }\end{array}$ \\
\hline
\end{tabular}


Quadro 2 - Síntese dos elementos da avaliação do processo de atendimento do Acolhimento com Avaliação e Classificação de Risco

(conclusão)

\begin{tabular}{|l|l|l|}
\hline $\begin{array}{l}\text { Dimensão } \\
\text { avaliada }\end{array}$ & \multicolumn{1}{|c|}{ Local avaliado } \\
$\begin{array}{l}\text { Assistência ao } \\
\text { usuário }\end{array}$ & $\begin{array}{l}\text { Recepção e } \\
\text { Classificação de Risco }\end{array}$ & $\begin{array}{l}\text { Resultados } \\
\text { a consulta de enfermagem na Classificação de Risco, } \\
\text { os profissionais, na maioria das vezes, faziam a escuta } \\
\text { qualificada. }\end{array}$ \\
\cline { 2 - 3 } & Classificação de Risco & $\begin{array}{l}\text { O enfermeiro realizava o exame físico superficial, de } \\
\text { acordo com a Queixa Principal do usuário. }\end{array}$ \\
\cline { 2 - 3 } & $\begin{array}{l}\text { Classificação de Risco e } \\
\text { Unidade de Emergência }\end{array}$ & $\begin{array}{l}\text { O preenchimento da ficha com a Queixa Principal } \\
\text { e o exame físico do usuário eram feitos de forma } \\
\text { adequada, e as informações colhidas eram registradas. }\end{array}$ \\
\cline { 2 - 3 } & Classificação de Risco & $\begin{array}{l}\text { O enfermeiro tinha autonomia para solicitar exames e } \\
\text { dar encaminhamentos. }\end{array}$ \\
\hline
\end{tabular}

Fonte: Elaboração própria.

\section{Discussão}

Em se tratando de um serviço hospitalar de emergência referência para a região do Recôncavo baiano, e considerando que a implantação da tecnologia do AACR nesse serviço foi concomitante a sua inauguração, a avaliação da estrutura e do processo é premente, porque se referem às condições oferecidas aos usuários e aos procedimentos realizados, além de subsidiarem a tomada de decisão pelos gestores ${ }^{(7)}$.

Para que o AACR seja desenvolvido eficazmente, além de profissionais capacitados e um protocolo efetivo que atenda as demandas da população, é necessária uma estrutura física apropriada, representada pela organização do espaço, dos recursos físicos, e materiais adequados cujos itens essenciais favoreçam os processos de trabalho ${ }^{(1,3)}$.

A avaliação da estrutura faz menção a todos os atributos materiais e organizacionais que são relativamente estáveis no setor, para proporcionar a assistência ${ }^{(10)}$. Foram identificadas deficiências nos setores da CR, recepção e emergência, em relação ao acesso do usuário à informação, conforto, dignidade e cortesia, além da estrutura física e recursos materiais.

Quanto à avaliação estrutural do AACR, no que diz respeito ao acesso à informação, observou-se que, em algumas áreas, a sinalização era deficiente, obrigando o paciente a procurar auxílio de funcionários para acessar outros espaços. Apesar de existirem placas indicativas da recepção, consultório da $\mathrm{CR}$, consultório médico, serviço social, sala de sutura, sala de gesso, observação masculina, feminina e pediátrica, sala de estabilização, sala de reanimação, posto de enfermagem, esses espaços não estavam sinalizados por cores. Desse modo, o MS sugere que a área do AACR seja organizada em dois eixos distintos: o vermelho, para o paciente grave ou com risco de morte, e o azul para o paciente aparentemente não grave. Cada eixo deve ser subdivido em áreas, também sinalizadas por cores, de acordo com as atividades nelas desenvolvidas ${ }^{(3)}$.

Um dado que chama a atenção na avaliação da estrutura física é que, apesar de a UE avaliada apresentar todas as dependências obrigatórias, conforme preconizado, observou-se áreas com disposições diferentes, e a sala de reanimação não estava bem localizada e nem sinalizada, o que poderia repercutir na garantia da rápida transferência dos pacientes graves. Em situações como uma parada cardiorrespiratória, insuficiência respiratória, crise convulsiva, dentre outras que necessitem de cuidado imediato, não se pode perder tempo no encaminhamento ou na procura de equipamentos, materiais e medicamentos necessários ao atendimento ${ }^{(5)}$. Tal deficiência assemelha-se à realidade de uma 
pesquisa realizada em uma UPA no sul do país, que aponta fragilidades em toda a estrutura física do serviço, contribuindo, assim, para a fragilidade dos processos de trabalho ${ }^{(10)}$.

Outro aspecto a ser destacado acerca da avaliação da estrutura, refere-se ao item conforto. Apesar de o mobiliário ter sido encontrado limpo e em bom estado de conservação, o número de cadeiras na recepção era insuficiente para acomodação dos usuários e seus acompanhantes, refletindo, assim, a superlotação e corroborando achados de outros estudos ${ }^{(9,11-12)}$ cujo conforto do usuário ou acompanhante foi o quesito avaliado de forma insatisfatória. Esse dado merece atenção, pois, sob a ótica da humanização, o acolhimento é primordial, embora melhorias no espaço físico, como poltronas confortáveis, áreas de entretenimento e descanso e afins, sejam onerosos para os gestores ${ }^{(12)}$.

Sobre a acomodação dos acompanhantes e o direito de permanecerem com os usuários que são atendidos, notou-se que nem sempre isso foi possível, ficando o acompanhamento restrito a alguns idosos e crianças. Essa fragilidade assemelha-se a outras realidades, em que a estrutura física foi um fator limitante ao acesso do acompanhante durante o período de espera e realização do atendimento, seja pela falta de salas apropriadas, pouca privacidade, ou, ainda, pela superlotação ${ }^{(1,13)}$.

É importante lembrar que o AACR tem interface com os dispositivos da Política Nacional de Humanização (PNH), inclusive quanto à ambiência e ao direito ao acompanhante ${ }^{(3)}$, quando estabelece a garantia de visita aberta com a presença do acompanhante e de sua rede social. Todavia, considera que devem ser respeitadas a dinâmica de cada unidade hospitalar e as peculiaridades das necessidades dos usuários. Desse modo, a unidade de emergência deve proporcionar aos visitantes uma recepção, lugares de espera e ambientes de escuta acolhedores e humanizados, para que possam aguardar confortavelmente pelas informações sobre o estado clínico dos pacientes, quando a presença deles não for possível junto ao leito ${ }^{(3,10)}$.
A avaliação da dimensão dignidade e cortesia envolve o direito do indivíduo de ser tratado como pessoa e não apenas como paciente ${ }^{(9)}$. Quanto a essa avaliação, destaca-se a presença de uma caixa de sugestões na recepção, para recolher a opinião dos usuários. É importante que gestores e profissionais tenham conhecimento da percepção do usuário sobre o serviço. Estudo aponta que é imprescindivel a obtenção de informações dos usuários que utilizam o AACR, pelo fato de participarem de todo o processo de construção e validação ${ }^{(14)}$.

Os resultados da avaliação dos recursos materiais disponíveis para o AACR evidenciaram que, apesar do tempo de implantação do AACR na instituição, ainda faltavam materiais preconizados pelo $\mathrm{MS}^{(3)}$ na sala de CR, tais como: monitor multiparamétrico, aparelho de ECG e instrumentos para reanimação cardiopulmonar. O déficit em relação aos recursos materiais, expresso pela ausência ou precariedade desses materiais, pode, inclusive, influenciar na superlotação da unidade, pois impacta de forma negativa no bom andamento das ações a serem realizadas $^{(15)}$.

A análise das dimensões da avaliação da estrutura do serviço estudado evidenciou que a adequação do ambiente e as intervenções focadas na estrutura e na organização da unidade de emergência são necessárias para o atendimento eficiente do paciente na $\mathrm{CR}^{(16)}$. A avaliação do processo inclui toda a assistência que a equipe de saúde, enquanto provedores de atenção, presta a seus pacientes, e a habilidade com a qual a exerce ${ }^{(17)}$. A apreciação dos elementos processuais visualizados no Quadro 2 também denotam algumas fragilidades.

A dimensão privacidade, avaliada durante o atendimento na sala de CR e na UE, relaciona-se com a condução do atendimento em condições que protejam a privacidade e não exponham a pessoa a situações constrangedoras ${ }^{(9)}$. Observou-se que o usuário, quando examinado, na maioria das vezes não foi exposto a outras pessoas que não os profissionais de saúde. No entanto, a porta da sala da CR frequentemente encontrava-se aberta, permitindo a entrada 
indiscriminada de outros profissionais e até mesmo de usuários que continuavam a chegar em busca de atendimento.

Outros estudos avaliaram a privacidade do atendimento na UE. Um deles constatou um grau de insatisfação, na opinião dos usuários ${ }^{(9)}$; o outro, revelou que os enfermeiros discordaram que a classificação de risco proporcionasse o acolhimento e a privacidade do paciente ${ }^{(16)}$. Neste sentido, manter a privacidade diante de circunstâncias de fragilidade física, emocional e/ou psíquica torna-se um direito individual dos usuários, que contempla situações relacionadas à privacidade nas consultas, ao respeito à dignidade do sujeito e à proteção da sua intimidade. Desse modo, os profissionais de enfermagem têm a responsabilidade ética e o compromisso de garantir a privacidade e a confidencialidade dos pacientes ${ }^{(10,18)}$.

Houve destaque para a avaliação do estado clínico do paciente, no que se refere ao item definição na prioridade do atendimento. O caminho percorrido pelo usuário dentro do serviço estudado condiz com o que é preconizado pelo $\mathrm{MS}^{(3,5)}$. Ao chegar à UE, os pacientes preenchem a ficha na recepção e aguardam o atendimento do enfermeiro na sala de CR. No entanto, os casos mais graves são, de fato, priorizados, isto é, os pacientes classificados como vermelhos ou mesmo aqueles sem avaliação prévia, que chegam ao hospital sob o risco iminente de morte, recebem atendimento imediato. Os demais casos são classificados conforme sua gravidade clínica e aguardam o atendimento médico.

Esse resultado reafirma o objetivo da triagem em serviços internacionais e nacionais quanto a determinar e classificar os pacientes rapidamente em ordem de urgência com base na necessidade clínica $^{(16)}$. Considera-se, portanto, que essa metodologia promove menor agravo à saúde dos usuários, pois facilita a classificação e a orientação do fluxo de pacientes, de tal modo que o mais grave é priorizado em relação ao menos grave $^{(10)}$.

Estudo internacional também reconhece que pacientes em fase aguda da doença enfrentam atrasos inaceitáveis no atendimento inicial em
SE, devido à falta ou à classificação de risco inadequada $^{(19-20)}$. Na tentativa de sanar problemas desse tipo, os sistemas de saúde de diferentes países têm criado distintas formas de triagem classificatórias de risco ${ }^{(19)}$. No cenário avaliado, pôde ser observada a utilização de um protocolo de CR institucional, que orientava a decisão do enfermeiro na priorização de atendimento dos usuários em quatro níveis. A utilização de protocolos pré-estabelecidos no AACR tem sido necessária e deve atender às demandas da população e respeitar a individualidade do paciente em todos os aspectos. Assim, oferece respaldo legal para a tomada de decisão do enfermeiro da $\mathrm{CR}^{(15-16)}$.

Ao chegar ao serviço de emergência, o acesso dos pacientes ao setor de CR deve ser imediato ${ }^{(21)}$. Assim, os tempos de espera dos usuários no processo de atendimento do AACR são atributos importantes a serem avaliados. As atividades da CR ocorrem em um ambiente sob pressão do tempo, algumas vezes com insatisfação dos usuários, devido ao tempo de espera pelo atendimento ${ }^{(22)}$. Neste estudo, observou-se que o tempo de espera do usuário para ser classificado era mensurado na chegada (preenchimento da ficha) e o usuário era comunicado sobre o período estimado em que devia aguardar o atendimento médico. O recomendado é que esse tempo de espera não ultrapasse, na categoria de menor urgência, 120 minutos $^{(21)}$. Contudo, é notório que esse tempo, em muitos casos, é extrapolado e os usuários, em sua maioria, não são avisados, tampouco reavaliados, a não ser que haja um agravamento do quadro clínico, que sempre é priorizado.

A reclassificação dos pacientes ao longo do período de espera para atendimento médico é de suma importância, pois, muitas vezes, pode ocorrer uma deterioração da sua condição clínica em decorrência da demora ${ }^{(10)}$. Há imperativa necessidade de o enfermeiro estar preparado para classificar e reclassificar, quando necessário, a prioridade de atendimento do usuário ao longo do período de espera. Para tanto, sua avaliação deve ser cíclica ${ }^{(21)}$, isto é, com contínuo planejamento e reavaliação dos usuários, 
independentemente do tempo em que estão à espera do atendimento médico.

Ainda concernente ao processo de avaliação do AACR, constatou-se, na observação, que a CR era realizada diariamente por apenas um enfermeiro. No entendimento dos enfermeiros, havia sobrecarga de trabalho na CR, apesar de atender todas as pessoas que procuravam o setor. A superlotação da emergência foi observada neste estudo, sendo mais evidente nos horários de pico do serviço, entre $10 \mathrm{~h}$ e $18 \mathrm{~h}$.

Estudos desenvolvidos em serviços de emergência demonstraram que o quantitativo insuficiente de enfermeiros na CR, diante da excessiva demanda e dos conflitos decorrentes da priorização de atendimento, são fatores geradores de desgaste e sobrecarga emocional para esses profissionais $^{(13,23)}$. Nesse sentido, torna-se necessário repensar o dimensionamento de pessoal, e também o protocolo Estadual de Classificação de Risco do Estado da Bahia, que tem recomendado a participação do técnico de enfermagem na CR. Dentre suas atribuições, destaca-se o atendimento ao usuário de forma acolhedora, recepção para o acolhimento e identificação de possíveis situações de urgência e de sinais vitais, observação e encaminhamento imediato do paciente para a sala de classificação de risco, quando julgar necessário ${ }^{(8)}$.

Em relação ao processo de avaliação da assistência prestada ao paciente no AACR, observou-se que, durante a chegada do usuário, no preenchimento da ficha pela recepção e durante a consulta de enfermagem na CR, os profissionais, na maioria das vezes, faziam a escuta qualificada. Pôde-se observar neste estudo que a ação de acolhimento dos usuários era realizada quase que exclusivamente na recepção e na sala da CR, descaracterizando a proposta preconizada pelo MS, que considera que o acolhimento ao usuário em SE e em todos os espaços da emergência pode ser realizado por qualquer profissional de saúde treinado para esse fim $^{(3)}$, sendo apenas a CR de competência do enfermeiro. Este profissional é também responsável pela tomada de decisão acerca do nível de prioridade clínica ${ }^{(1)}$.
Para o desenvolvimento da CR, o enfermeiro, além de utilizar a escuta da situação/queixa, do breve histórico, anamnese, realiza o exame físico e verifica os sinais vitais. Observou-se que as informações colhidas eram registradas adequadamente e o exame físico do usuário era feito de forma sucinta, de acordo com a queixa principal. Assim, na avaliação da situação clínica do paciente, a escuta das queixas aliada ao compromisso de dar respostas às necessidades de saúde trazidas por ele, consiste em habilidade importante dos enfermeiros no processo de $\mathrm{CR}^{(16)}$.

Outro item avaliado no AACR foi o processo de trabalho do enfermeiro na $\mathrm{CR}$, no que concerne à autonomia para solicitar exames e dar encaminhamentos. Para esses casos, observou-se que, na chegada de um paciente com queixa de dor torácica, com ou sem irradiação, sudorese, palpitação ou qualquer outro sinal ou sintoma sugestivo de doença coronariana aguda, o enfermeiro, algumas vezes, fazia solicitação de realização de ECG antes mesmo de uma avaliação médica, a fim de agilizar o atendimento e priorizá-lo, se necessário. Esse achado é apoiado por estudo internacional, quando revela que os enfermeiros do SE devem saber antecipar os cuidados e agilizar as intervenções apropriadas para responder ao déficit fisiológico apresentado pelo paciente. Além disso, o enfermeiro possui um conjunto de competências baseadas no objetivo de atender às necessidades do paciente e otimizar os resultados ${ }^{(24)}$.

Algumas dimensões da avaliação do processo de atendimento do AACR constituem-se em desafios, a exemplo do encaminhamento dos usuários após a CR. Observou-se que nem todos que passavam pelo AACR eram direcionados para o atendimento médico. Quando classificados como azul (não urgentes), eram encaminhados para o atendimento no ambulatório do próprio hospital ou orientados a procurar uma unidade básica de saúde (UBS) ou outra instituição de saúde.

Considerando as recomendações do MS, numa situação de baixa gravidade, o usuário deve ser referenciado/contrarreferenciado para outro ponto da rede de atenção, a fim de garantir 
o atendimento ${ }^{(3,5)}$. Entretanto, observou-se que o encaminhamento realizado pelos enfermeiros deste estudo era informal, e muitos usuários eram direcionados a outras portas de entrada do sistema de saúde sem a ficha de referência ou contrarreferência. Outro estudo aponta que os mecanismos de referência e contrarreferência dentro da rede são frágeis, e essa ocorrência interfere no atendimento integral do usuário. Isso explica, em parte, a dificuldade de encaminhamento dos pacientes que necessitam de cuidados de baixa complexidade para a UBS mais próxima ${ }^{(12)}$. Ademais, contribui para que o paciente retorne diversas vezes à emergência sem a solução do seu problema, sobrecarregando-a ${ }^{(10,13)}$.

É válido lembrar que a Resolução no 2.077/2014, do Conselho Federal de Medicina, que dispõe sobre a normatização do funcionamento dos SE e propõe como obrigatória a implantação do AACR para atendimento nos serviços, estabelece que todo paciente que tiver acesso ao SE deverá, obrigatoriamente, ser atendido por um médico, não podendo, sob nenhuma justificativa, ser dispensado ou encaminhado a outra unidade de saúde por outro profissional que não o médico ${ }^{(20)}$.

Outra observação é que pode ocorrer a recusa de alguns pacientes a serem encaminhados a outros serviços. Nesse caso, a instituição não pode esquivar-se do atendimento, uma vez que o AACR não é excludente ${ }^{(3,7)}$. No serviço avaliado, o processo de trabalho estava estruturado para se desenvolver com base em ações que priorizavam os casos de maior gravidade para o atendimento. Um estudo de revisão sistemática desenvolvido com pesquisas internacionais identificou vários motivos pelos quais os pacientes optavam por acessar o SE. Dentre eles, destacavam-se: falta de acesso e confiança na atenção primária; percepção de urgência, ansiedade e o valor de reafirmação de serviços baseados em emergência; conveniência (localização, não ter que marcar consulta e horário de funcionamento) e fatores individuais do paciente (por exemplo, custo).

Considera-se que a investigação é limitada, pelo fato de a avaliação ser composta por atributos da estrutura e do processo de atendimento do AACR, não incluindo, portanto, a avaliação dos resultados da implantação do AACR. Um dos aspectos considerados na avaliação dos resultados tem sido a satisfação dos usuários ${ }^{(9)}$. No entanto, destaca-se que a avaliação do serviço de saúde pelo paciente pode ser influenciada pela estrutura e pela forma como é assistido pelos profissionais de saúde. A não utilização de um instrumento validado para a avaliação das dimensões, estrutura e processo, que retrate também a realidade dos diferentes serviços de emergências do Brasil, pode também ser considerada como limitante para o entendimento das fragilidades encontradas na CR. Daí a necessidade de novos estudos que avaliem a prática da CR em serviços de emergência, com vistas a contribuir para a resolução das fragilidades identificadas no presente estudo e qualificar o atendimento aos usuários.

\section{Conclusão}

A avaliação do AACR no SE da instituição estudada revelou avanços e potencialidades, bem como fragilidades e desafios a serem superados. Dentre os aspectos mais relevantes, destaca-se: a unidade de emergência é referência na região para o atendimento de diversas especialidades; o funcionamento do AACR é ininterrupto; e o enfermeiro é o profissional responsável para acolher, avaliar e classificar o risco dos pacientes que procuram o serviço. Ele é orientado por um protocolo próprio, direcionador, estratificado em quatro cores, atendendo a um critério de ordem que caracteriza as prioridades de acordo com a gravidade clínica do usuário.

A estrutura física e os recursos materiais atendem grande parte do que é preconizado pelo Ministério da Saúde, assim como o fluxo de atendimento. Entretanto, existem lacunas no tempo de espera estimado, o qual, muitas vezes, é extrapolado, além de ausência formal de referência e contrarreferência que interferem no atendimento integral do usuário no AACR.

Concernente às fragilidades, destacam-se: falta de sinalização dos espaços por cores e a integração entre os eixos; escassez de recursos 
para acomodação de acompanhantes; e ausência de alguns materiais na classificação de risco. As ações de acolhimento e a escuta qualificada dos usuários são realizadas quase que exclusivamente na sala da CR, descaracterizando a proposta preconizada pelo MS.

Assim, o quadro observado na emergência avaliada mostra a necessidade de investimentos tecnológicos para melhoria na qualidade dos serviços prestados, visto que os recursos materiais nele dispostos ainda não alcançaram o que é preconizado como ideal. O AACR deve ser entendido como estratégia dentro da instituição e sua efetividade sempre reavaliada, garantindo, assim, a segurança do paciente.

O estudo permitiu concluir-se que a estrutura e o processo do acolhimento com classificação de risco em uma unidade de emergência precisa de adequação do espaço físico e do suprimento total de recursos para cumprir o que é proposto pelo Ministério da Saúde.

\section{Colaborações:}

1 - concepção, projeto, análise e interpretação dos dados: Ana Paula Santos de Jesus e Guiomar Rocha Pimentel Pimenta Rodrigues;

2 - redação do artigo e revisão crítica relevante do conteúdo intelectual: Ana Paula Santos de Jesus, Guiomar Rocha Pimentel Pimenta Rodrigues, Larissa Oliveira de Jesus, Meiry Fernanda Pinto Okuno e Ruth Ester Assayag Batista;

3 - aprovação final da versão a ser publicada: Ana Paula Santos de Jesus e Guiomar Rocha Pimentel Pimenta Rodrigues.

\section{Referências}

1. Inoue KC, Murassaki ACY, Bellucci Júnior JA, Rossi RM, Martinez YDÉ, Matsuda LM. Acolhimento com classificação de risco: avaliação da estrutura, processo e resultado. Rev Min Enferm [Internet]. 2015 [cited 2017 Apr 10];19(1):13-20. Available from: http://www.reme.org.br/artigo/detalhes/982

2. Machado CV, Lima LD, O'Dwyer G, Andrade CLT, Baptista TWF, Pitthan RGV, et al. Gestão do trabalho nas Unidades de Pronto Atendimento: estratégias governamentais e perfil dos profissionais de saúde. Cad Saúde Pública [Internet]. 2016 [cited 2017 May 12];32(2):e00170614. Available from: http://www.scielo.br/scielo.php?script $=$ sci_ arttext\&pid=S0102311X2016000200704\& $\operatorname{lng}=\mathrm{pt} \& \ln \mathrm{ln}=\mathrm{pt}$

3. Brasil. Ministério da Saúde. Secretaria-Executiva. Núcleo Técnico da Política Nacional de Humanização. Humaniza SUS: acolhimento com avaliação e classificação de risco: um paradigma ético-estético no fazer em saúde. 2a ed. [Internet]. Brasília; 2004. (Série B. Textos Básicos de Saúde). [cited 2017 Apr 21]. Available from: http:// www.saude.sp.gov.br/resources/humanizacao/ biblioteca/pnh/acolhimento_com_avaliacao_e_ classificacao_de_risco.pdf

4. Anziliero F, Soler BED, Silva BA, Tanccini T, Beghetto MG. Sistema Manchester: tempo empregado na classificação de risco e prioridade para atendimento em uma emergência. Rev Gaúcha Enferm [Internet]. 2016 dez [cited 2017 Apr 15];37(4):e64753. Available from: file:///C:/Users/ guigu/Desktop/Artigos\%20AACR/0102-6933rgenf-1983-144720160464753.pdf

5. Brasil. Ministério da Saúde. Portaria GM/MS $\mathrm{n}^{\circ} 2.048$, de 5 de novembro de 2002. Aprova o Regulamento Técnico dos Sistemas Estaduais de Urgência e Emergência [Internet]. Brasília; 2002 [cited 2017 Apr 10]. Available from: http:// bvsms.saude.gov.br/bvs/saudelegis/gm/2002/ prt2048_05_11_2002.html

6. Brasil. Ministério da Saúde. Portaria $\mathrm{n}^{\circ} 28$, de 8 de janeiro de 2015. Reformula o Programa Nacional de Avaliação de Serviços de Saúde (PNASS) [Internet]. Brasília; 2015 [cited 2017 May 4]. Available from: http://bvsms.saude.gov.br/bvs/ saudelegis/gm/2015/prt0028_08_01_2015.html

7. Bellucci Júnior JA, Vituri DW, Versa GLGS, Furuya PS, Vidor RC, Matsuda LM. Acolhimento com classificação de risco em serviço hospitalar de emergência. Avaliação do processo de atendimento. Rev enferm UERJ. 2015 jan/fev;23(1)82-7. DOI: http://dx.doi.org/10.12957/reuerj.2015.4976

8. Bahia Secretaria de Saúde do Estado. Protocolo Estadual de Classificação de Risco [Internet]. Salvador; 2014 [cited 2017 May 4]. 54 p. Available from: http://www.saude.ba.gov.br/wp-content/ uploads/2017/06/protocolo_classificacaoderisco_ jun_2017.pdf 
9. Mendes ACG. A delicadeza esquecida: avaliação da qualidade das Emergências. Recife: EdUFPE; 2010.

10. Hermida PMV, Nascimento ERP, EchevarríaGuanilo ME, Brüggemann OM, Malfussi LBH. User embracement with risk classification in an emergency care unit: an evaluative study. Rev Esc Enferm USP. 2018;52:e03318. DOI: http://dx.doi. org/10.1590/s1980-220x2017001303318

11. Souza TH, Andrade SR. Embracement with risk classification: an indicator of the emergency demand on a hospital service. Cogitare Enferm [Internet]. 2014 [cited 2017 Apr 10];19(4):643-50. Available from: http://revistas.ufpr.br/cogitare/ article/view/35941/23932

12. Costa MAR, Versa GLGS, Bellucci Júnior JA, Inoue KC, Sales CA, Matsuda LM. Acolhimento com Classificação de Risco: Avaliação de Serviços Hospitalares de Emergência. Esc Anna Nery [Internet]. 2015 jul-set [cited 2017 Apr 25];19(3):491-7. Available from: http://www.scielo. br/pdf/ean/v19n3/1414-8145-ean-19-03-0491.pdf

13. Hedlund ACB, Ilha CB, Hoffmann IC, Krusche JB, Pimenta LF, Braz MM. Percepção de profissionais sobre acolhimento com classificação de risco no centro obstétrico. Saúde (Santa Maria) [Internet]. 2015 jul/dez [cited 2017 Feb 25];41(2):149-60. Available from: https://periodicos.ufsm.br/ revistasaude/article/view/15059

14. Oliveira JLC, Gatti AP, Barreto MS, Bellucci Junior JA, Góes HLF, Matsuda LM. Acolhimento com classificação de risco: percepções de usuários de uma unidade de pronto atendimento. Texto Contexto Enferm. 2017;26(1):e0960014. DOI: http:// dx.doi.org/10.1590/0104-07072017000960014

15. Prudêncio CPG, Monteiro RAN, Ribeiro BCM, Gomes MSM, Manhães LSP. Percepção de enfermeira(o)s sobre acolhimento com classificação de risco no serviço de pronto atendimento. Rev baiana enferm. 2016 [cited 2017 Feb 20];30(2):1-10. Available from: https://portalseer.ufba.br/index. php/enfermagem/article/view/14917/pdf_46

16. Duro CLM, Lima MADS, Weber LAF. Opinião de enfermeiros sobre classificação de risco em serviços de urgência. REME rev min enferm. 2017;21:e1062. DOI: http://www. dx.doi.org/10.5935/1415-2762.20170072
17. Rocha AD, Sousa CPC, Pedraza DF, Queiroz D. Atenção básica à saúde: avaliação de estrutura e processo. Rev Adm Saúde [Internet]. 2012 [cited 2017 Feb 25];14(55):71-9. Available from: www.cqh.org.br/portal/pag/anexos/baixar. php?p_ndoc $=276 \&$ p_nanexo $=321$

18. Soares NV, Dall'Agnol CM. Privacidade dos pacientes: uma questão ética para a gerência do cuidado em enfermagem. Acta paul enferm [Internet]. 2011 [cited 2017 May 16];24(5):683-8. Available from: http://www.lume.ufrgs.br/ handle/10183/38500

19. Schuetz P, Hausfater P, Amin D, Haubitz S, Fässler L, Grolimund E, et al. Optimizing triage and hospitalization in adult general medical emergency patients: the triage project. BMC Emerg Med. 2013 jul;13:12. DOI: 10.1186/1471-227X-13-12

20. Sunyoto T, Vander Berg R, Valles P, Gutierrez R, Ayada L, Zachariah R, et al. Providing emergency care and assessing a patient triage system in a referral hospital in Somaliland: a cross-sectional study. BMC Health Serv Res. 2014 Nov 6;14:532. DOI: 10.1186/s12913-014-0531-3.

21. Conselho Federal de Medicina. Resolução no 2.077, de 24 de julho de 2014. Dispõe sobre a normatização do funcionamento dos Serviços Hospitalares de Urgência e Emergência, bem como do dimensionamento da equipe médica e do sistema de trabalho. Brasília; 2014 [Internet]. [cited 2017 Apr 17]. Available from: https://www. legisweb.com.br/legislacao/?id=274741

22. Carter EJ, Pouch SM, Larson EL. The relationship between emergency department crowding and patient outcomes: a systematic review. J Nurs Scholarsh. 2014 [cited 2019 Aug 18];46(2):106-15. Available from: https://sigmapubs.onlinelibrary. wiley.com/doi/abs/10.1111/jnu.12055

23. Hitchcock M, Gillespie B, Crilly J, Chaboyer W. Triage: an investigation of the process and potential vulnerabilities. J Adv Nurs. 2013 [cited 2019 Aug 18];70(7):1532-41. Available from: https://onlinelibrary.wiley.com/doi/abs/10.1111/ jan.12304

24. Cypress BS. The emergency department: experiences of patients, families and their nurses. Adv Emerg Nurs [Internet]. 2014 [cited 2019 Aug 
18];636(2):164-76. Available from: https://www. ncbi.nlm.nih.gov/pubmed/24785669

25. Coster JE, Turner JK, Bradbury D, Cantrell A. Why do People Choose Emergency and Urgent Care Services? A Rapid Review Utilizing a Systematic Literature Search and Narrative Synthesis. Acad
Emerg Med. 2017 Sep;24(9):1137-49. doi: 10.1111/ acem. 13220

Recebido: 1 de outubro de 2018

Aprovado: 9 de setembro de 2019

Publicado: 14 de novembro de 2019

A Revista Baiana de Enfermagem utiliza a Licença Creative Commons - Atribuição-NãoComercial 4.0 Internacional. https://creativecommons.org/licenses/by-nc/4.0/ Este artigo é de acesso aberto distribuído sob os termos da Licença Creative Commons (CC BY-NC). Esta licença permite que outros remixem, adaptem e criem a partir do seu trabalho para fins não comerciais. Embora os novos trabalhos tenham de lhe atribuir o devido crédito e não possam ser usados para fins comerciais, os usuários não têm de licenciar esses trabalhos derivados sob os mesmos termos. 